\title{
Evaluating library services - best practice initiatives in Australian university libraries
}

\author{
Helen King \\ Associate Librarian \\ La Trobe University Library \\ Victoria, Australia \\ belen.king@latrobe.edu.au
}

\section{Introduction}

Collection and use of data in the planning and management of library activities, and the measurement of performance of these activities has a long history. In recent years, with the automation of library functions and the shift to a networked information environment, the potential sources and types of data that can be collected have increased, at an almost exponential rate. This has resulted in the need for libraries to refocus on data collection, question their current activities, and rationalise efforts to ensure that the range of data being collected is relevant, sustainable and meets specific objectives. In other words the reasons for collection are clearly understood by all, and can be justified in terms of their role in the monitoring and improvement of library performance. Hiller and Self (2004) provide a useful historical summary, tracing the evolution of data collection and its application in improving library performance. The authors contend that a key catalyst in the evolution of collecting data for improvement was the introduction of strategic planning in libraries during the 1980s. This resulted in the implementation of planning and improvement frameworks, which identified overall library objectives, action plans to meet those objectives, their implementation, the monitoring and evaluation of results through measurement activities, and the consequent introduction of new actions to improve performance.

This evolution of data collection for measurement purposes has now reached a stage where performance measures are being grouped into three main categories - 
inputs, outputs and, increasingly, outcomes (or impacts). Examples of inputs are size of collections, size of budgets and staffing profiles; examples of outputs include use of collections, number of reference questions answered and number of information literacy classes held. Outcomes, on the other hand, are more complex and have been broadly defined as "what impact the library has on the life of its clientele" (Hiller and Self, 2004, p.140) and "the ways in which library users are changed as a result of their contact with the library's resources and programs" (ACRL, 2000). This shift in the measurement focus is being particularly driven in the United States, with the many accreditation agencies now moving to outcomes based assessment. However, as Cullen (2002) observes, "outcomes have proved to be a more difficult area of evaluation, and there is no work to date on standards for outcomes". Hiller and Self $(2004$, p.2) in discussing recent deliberations of the ARL Committee on Statistics and Measurement, note the ARL comments that "it is only through using a wide variety of measures that one can hope to get a full and accurate reading of the library's activities". The ARL New Measures Initiative was established to explore data collection measures that would "go beyond traditional input/output measures, to capture the use and impact of libraries". Eight areas of interest have been identified by the ARL initiative - user satisfaction, market penetration, ease and breadth of access, library impact on teaching and learning, library impact on research, cost effectiveness of library operations and services, library facilities and space and organisational capacity (ARL, 1999).

Hernon and Dugan (2003) further note the need to recognize that the measures utilised will differ according to the perspective of who wants to know. They contend there are four perspectives which need to be considered -

1. library centred - the library's perceived service priorities and its success in meeting these priorities as defined by input, output and performance measures

2. institution centred - the output and performance measures used to demonstrate the library's contribution to accomplishing the institution's mission

3. user or customer centred - the comparison of user expectations with service performance, the gap between expectations and performance and the data that will lead to improved service delivery

4. stakeholder centred - measures will include elements from the above three but also new requirements for demonstrating institutional effectiveness and efficiency. The focus shifts to "what happened because of the outputs" and introduces the concept of outcomes assessment. In the case of libraries, an example would be "how does the library's information literacy program contribute to the knowledge and skills that students should display by the time of graduation". The authors argue that the impact of outcomes assessment 
is only starting to unfold and will alter institutional and organisational (e.g. library and academic programs) cultures over time.

The challenge for library researchers will be to develop, as Hernon and Dugan note (2003, p.2) "a universal set of measures and data reporting methods from which we can select, given the perspective we are trying to address."

\section{Terminology}

The evolving nature of the measurement debate requires some clarification of the terminology used in this more practically oriented paper, which describes the initiatives in Australian academic libraries. Here, the definitions of Hernon and Dugan are followed, namely

Assessment is a set of initiatives the institution takes to review the results of its actions, and make improvements

Evaluation is narrower, and produces data to improve service and to ensure the results conform to the stated mission, goals and objectives

This paper focuses on evaluation activities which, unlike the concept of assessment, and more specifically outcomes assessment, are well developed and understood and have been used over a long period of time.

\section{The Australian Perspective}

Australian university libraries have been implementing performance measurement, benchmarking and quality improvement in library and information services for many years. In the strict sense of the definitions outlined above, these have been primarily relatively simple evaluation activities. That is, the gathering of data to improve services as identified in the library's mission, goals and objectives.

There are examples, however, of some libraries moving from simple evaluative activities to a more holistic approach to library assessment, as defined above, experimenting with frameworks developed in the business world. A leader in the development of a whole of library performance measurement framework is the University of Wollongong Library in New South Wales. This library adopted the Australian Quality Council's Australian Business Excellence Framework in 1994 and developed a Performance Indicator Framework which links performance indicators and measures to the library's goals, critical success factors and stakeholder needs and expectations (McGregor, 2000; McGregor, 2003). It was the first library to win an Australian Business Excellence Award.

Other business frameworks being adopted by libraries include the internationally used Balanced Scorecard. Cribb (2003) describes the experience of Bond University Library (Queensland) in applying this approach. 


\section{Council of Australian University Librarians (CAUL) Initiatives}

In Australia, national co-ordination and encouragement of collaborative initiatives between university libraries, including the development of performance measurement and benchmarking is facilitated by CAUL, the Council of Australian University Librarians, primarily through its Best Practice Working Group. Significant achievements in this area which have been achieved through CAUL initiatives are

1. the annual collection of statistical information which enables comparison of key library input and output measures, and the ongoing revision and updating of the range of statistics that are collected by university libraries.

2. the development of three performance indicator kits in 1995 which were intended to provide standard instruments to be used by libraries in monitoring their own performance, and sharing this performance information with other libraries

a. Library/clientele congruence indicator

b. Document delivery performance

c. Proportion of sought material obtained at time of visit (Materials availability)

\section{Library/clientele Congruence Indicator}

This indicator has now been largely overtaken by the recent development of a national customer satisfaction survey instrument which is discussed later in this paper.

\section{Document Delivery Performance Indicator}

The document delivery performance indicator enables libraries to assess the time taken to process and fill document delivery requests on behalf of clients, and evaluate the efficiency and effectiveness of their document delivery practices. The kit has undergone several updates since 1995.

\section{Materials Availability Performance Indicator}

Materials availability is defined as the proportion of material sought by the user which is available in the library at the time it is sought. Information from the survey provides data on availability and the reasons for non availability. Although designed in 1995, when the print environment predominated, the instrument continues to be used. CAUL is now looking at developing an electronic materials availability indicator, which recognises the impact of wanted material in electronic form, and the emergence of virtual (as well as physical) visits to the library.

CAUL has developed a Best Practice Database on the CAUL website ${ }^{1}$ which enables the loading of individual library results for Document delivery performance and Materials availability surveys, and also provides access to results of 
the customer satisfaction surveys. Results can then be compared with both a specific library's previous performance, and the performance of other academic libraries in the database, providing both internal and external performance benchmarking opportunities.

3. the sponsorship of the Performance Measures for Reference Services Working Party established in 1994 to:

- identify performance measures and indicators currently used to evaluate reference services in Victorian academic libraries

- identify from the professional literature performance measures and indicators for evaluating reference services in academic libraries

The final report of the Working Party was published in $1998^{2}$ and summarises the indicators and measures used by undergraduate, postgraduate and academic staff at 8 Victorian universities, based on focus-group interviews conducted during 1996 and 1997.

4. the sponsorship of the project "Best Practice for Australian University Libraries" which received significant funding from the Australian government and resulted in the publication of two major reports published in 2000 "Guidelines for the Application of Best Practice in Australian University Libraries" 3 and the companion "Best Practice Handbook for Australian University Libraries"4. This project investigated and compiled current "best practice" activities within Australian academic libraries and compared these with activities in selected overseas countries. The term "best practice" used in the study encompassed the implementation of quality frameworks, and the use of benchmarking and performance measurement as tools for the continuous improvement of products, processes and services.

5. the development of best practice guides, such as the CAUL Principles for Library Services to Offshore Students. This is an aid to libraries in providing services to students enrolled in offshore campuses of Australian universities.

The CAUL website also provides the opportunity for university libraries to load or make reference to a range of their planning documents (such as building plans, strategic plans, workflow diagrams, skills audits and staff development plans) which would be of wider interest to the academic library community and assist in identifying best practice.

6. sponsoring the development and assisting in the administration of a national customer satisfaction survey instrument (developed by Rodski Behavioural Research and known as the Rodski survey). 


\section{The Rodski Customer Satisfaction Survey}

This is currently being used by 37 Australian and New Zealand university libraries, many now implementing it on a biennial basis. Survey results provide a guide for potential improvements in the areas of services, collections, facilities and library staff behaviours, based on the gap between how important the service is to the user and the user perception of how well the library is performing in that area. The survey includes a set of nearly 40 core statements or variables, common to all participants. There is also the opportunity for a library to add a small number of customised statements specific to local needs. Respondents to the survey are asked to rank each of the statements according to the level of importance to them (on a scale of 1 to 7 ), and then to rank the library's perceived performance, again on a 1 to 7 scale and from the respondent's perspective. The detailed analysis provides ranked lists of the statements according to both importance and performance, and subsequently a ranked list of statements according to the gap between importance and performance, the assumption being that the larger the gap, the greater the level of dissatisfaction, and the greater the need to introduce improvement in that particular area.

The statements or variables are grouped into the broad categories - Communication, Service Quality, Service Delivery, Facilities and Equipment and Library Staff. Two further questions ask respondents to rate the overall quality of the library and their overall satisfaction with the library on a scale of 1 to 7. The quantitative results are further informed by free text comments which respondents provide in answer to 1 . Comments (including positive and negative) and 2. What is the one area we could improve to assist you?

Rodski Behavioural Research provides support for the analysis of the survey and has also developed a results website which enables comparison or benchmarking of individual library results from survey to survey, comparison against the means of all other participants in the database and also against the performance of individual participants. The CAUL Best Practice Working Group also surveyed the top performing libraries in the Rodski surveys to share ideas for improvement. La Trobe University Library has published the results of the two surveys so far conducted (in 2002 and 2004), on the library website ${ }^{5}$.

Ongoing work by CAUL includes:

1. the enhancement of the Rodski survey to include questions of relevance to students studying at a distance.

2. the development of a set of performance measures for digital research and information services. This work will build on the Performance Measures for Reference Services work, detailed above.

3. the development of an information literacy assessment instrument. 


\section{Other Benchmarking Activities}

Within Australia's 39 universities, formal sub groups have been established where there are commonalities of background and purpose. These sub groups are an avenue to explore and encourage collaboration and exchange of information between institutions and the further pursuit of common goals, one of which being to compete more effectively with the other universities. Such sectors include the Australian Technological Universities ATN (those universities which have come from a technological background), Innovative Research Universities Australia IRUA (universities established in the late 60 s to cater for the growing demand for tertiary education and usually located in outer suburban areas of capital cities), the Group of Eight universities GO8 (the oldest, most wealthy and heavily research oriented universities).

These sectors usually involve their libraries, who meet to discuss and share information about areas of common interest, and identify collaborative opportunities such as benchmarking of performance across a range of areas. For example, the IRUA group of libraries has been undertaking the benchmarking of a range of "processing" activities, the objective being to identify the best performers and look at best practice, with the aim of improving individual library performance.

\section{The Australian Higher Education Context}

The emphasis on qualitative and quantitative measurements of performance at the institutional level has taken on increasing importance in recent years with the establishment of a formal quality audit framework within the Australian higher education system. The Australian Universities Quality Agency (AUQA) was established in March 2000 to promote, audit, and report on quality assurance in Australian higher education ${ }^{6}$. This independent body is charged with conducting quality audits of self accrediting higher education institutions (primarily universities) on a five yearly cycle. The audits are aimed to be fair, rigorous and authoritative, and the audit reports of individual institutions are subsequently made public on the AUQA website.

AUQA does not impose an externally prescribed set of standards but rather uses as its primary starting point each institution's own objectives. The audits focus on examining the processes planned and in place by which the institution achieves its own defined objectives, the outcomes that are achieved and the monitoring regime that is in place to check and improve outcomes. Each university is expected to devise its own quality management system best suited to meet the specific needs of that university. This includes appropriate quantitative and qualitative measures and indicators.

AUQA does not rank institutions based on the audits, or produce any sort of league table, although inevitably, due to the public availability of the reports, institutional comparisons are made. Each audit is based on a self review conducted by 
the university itself which is reported in an institutional Performance Portfolio and submitted to AUQA. This is followed by an audit visit to the institution (usually 3 to 5 days) to test the content and the assertions in the portfolio. AUQA publishes a report on the audit approximately 3 months later, including commendations, affirmations (where AUQA affirms the improvement needs which have already been identified by the institutions) and recommendations. The university is then expected to produce an action plan showing how it will respond to the audit findings, specifically the recommendations. In the next audit round (approximately 5 years later), AUQA will review the institution's response to the first audit. In 2006, the first round of audits of Australian universities will be completed.

The audits include scrutiny of all areas of academic endeavour including the university libraries. Library specific sections of the AUQA audit reports published to date have been focused on assessing the available evidence for

- Library planning and evaluation processes

- How priorities (and funding) are determined

- Client satisfaction with services

- Effectiveness of services provided

- Consistency of services across campuses

- Adequacy of services for overseas students

- Benchmarking of services and best practice

\section{La Trobe University Library - A Case Study}

La Trobe University is located in Victoria, Australia. It is a multi campus university, with 7 campuses located in metropolitan (the largest campus) and regional Victoria. It caters for 26,000 students, of which 3,600 are international students and a further 1,200 are located overseas. The campus student populations range from nearly 18,000 at the Bundoora (Melbourne) campus to just 58 at the Mt Buller campus.

The University Library at La Trobe operates as "one library" with a campus library presence at 6 of the 7 campuses. The library has a collection of more than 1.6 million physical volumes, substantially augmented by scholarly information in electronic format, including access to more than 35,000 electronic journals and databases. Due to the disparity in size of the campus libraries and the geographic separation, the library has moved rapidly into electronic resources and multi-site licencing, aiming at parity of access to information regardless of the location of a La Trobe student or staff member. The print collection is also accessible to all students and staff through a fast and efficient intercampus loan and document delivery service. 
The University was formally audited by AUQA in September 2004. Preparation for the AUQA audit precipitated the establishment of a self review regime and the articulation of a quality management system at the institutional level. At La Trobe, quality assurance is devolved to a unit level planning and continuous improvement cycle. The University is committed to the benchmarking of these operations and to the continuous improvement of the outcomes of its activities in the light of appropriate measures and indicators of quality assurance and accountability.

In preparation for the audit and the compilation of the Performance Portfolio, each unit (all academic and administrative areas, including the university library) was expected to carry out a self review of activities, identifying the success (or otherwise) in achieving its objectives, targets, or quality improvement initiatives. Outcomes of the review were expected to inform unit strategic planning for the forthcoming year and also include a quality improvement plan for that year. Ongoing self review has now been mainstreamed within the University, with formal review cycles and designated responsibility for the monitoring of these reviews established from the institutional level (e.g. University Council) down to the unit level.

To assist in the self review process the University's Quality Office developed "Quality Management Checklists" for the administrative and academic areas of the University.

\section{Library Preparation for the Audit and the Self Review Process}

The University Library has a well established planning process which required little modification to incorporate the requirements of the initial self review. The Library Strategic Plan 2000 - 2004 was developed initially through an extensive process of consultation with staff across the campus libraries. In addition, there was consultation and input from the university community. In each year of the plan, over the August/September/October period and prior to the annual internal library budget allocation, senior library staff review directions, and identify and refine priorities for the following year in a workshop environment. This process incorporates prior input from a wide range of library staff through campus/work area meetings held during the year.

The pre AUQA self review was incorporated into the above planning process. The library developed its own self review checklist incorporating appropriate elements from both the academic and administrative checklists. As part of the review, the library documentation (e.g. policy statements) which supports the evidence presented in the self review was also identified and included. The broad areas which the self review addressed included 
1. Planning and management (planning, budgeting, communication)

2. Staff (staff development, position descriptions, staff induction, leave management, performance management)

3. Stakeholders and standards (client service training, client feedback, service standards)

4. Policies and processes (development, documentation, communication, review)

5. Community engagement

6. Outcomes (performance indicators and outcome measures)

7. Response (strengths and shortcomings, opportunities for improvement, actions)

The template used for the library self review is included as Appendix 1.

The section 'Outcomes' in the Self Review Checklist revealed some opportunities for improvement for the library. Responses were required for the following questions

1. How do you know you are achieving your objectives? What performance indicators or outcome measures do you use?

2. Are these measures closely matched to the objectives?

3. Do you seek customer feedback from internal and external customers?

Analysis of these revealed a gap in library evaluation activities. Activities being undertaken at the time were limited, somewhat adhoc in their timing and only loosely linked to the library's strategic objectives. As a consequence and as part of the quality improvement plan, the library identified the need to take a more systematic approach, expand the performance measurement and benchmarking of key library areas and further develop the suite of service standards.

In the absence of a universally accepted set of standard outcome measures (as previously defined) for academic libraries, La Trobe is identifying a mix of input, output and outcome indicators (both qualitative and quantitative), in order to measure performance in achieving its strategic objectives, and to identify areas for improvement. These measures, in the context of a whole of library assessment set, are incomplete, imprecise and continually evolving at this stage. There are difficulties in defining a set that is manageable (avoiding the danger of 'analysis paralysis'), meaningful at the "whole of library" strategic level and persuasive, at the institutional level, in demonstrating the library's contribution to the outcomes or impacts of the parent university's endeavours as a whole.

The measures currently in use and under development by La Trobe University Library are listed in Appendix 2. 


\section{Future Challenges}

The task for Australian university libraries will be to broaden the current suite of evaluative activities that all are undertaking, to a greater or lesser extent, and move to more outcomes focussed activities. Some progress in this direction has already been described earlier in this paper. Pressure to demonstrate the value that academic libraries add to the Australian higher education system is likely to intensify as the proportion of government funding available to universities continues to contract, and universities are required to generate more and more resources from other sources.

Similar scenarios are being played out in the international arena. For example, Lakos $(1998,1999)$, in describing the North American environment, outlines the culture of assessment that must be developed "in order for libraries and librarians to be able to work in an environment where decisions are based on facts, research and analysis, and services are planned and delivered in order to maximise positive outcomes and impacts for the library clients". He contends that, despite the long history of data collection by libraries, assessment activities are still not routinely integrated into library operations, and are rarely applied systematically with appropriate follow-through and evaluation.

Lindauer (1998) documents the influence of the outcomes assessment movement of accreditation agencies in the United States, which now require that institutions provide evidence of their contributions to desired educational outcomes. In a very comprehensive paper, she identifies "important institutional outcomes to which academic libraries contribute, describes specific performance indicators whose measures of impacts and outputs provide evidence about progress and achievement, and offers a conceptual framework of assessment domains for the teaching-learning library". The schema provided however is very large and complex and is not practical to be implemented in its entirety.

The ultimate challenge will be to select key indicators and associated assessment activities which clearly demonstrate "what impact the library has on the life of its clientele" (Hiller and Self, 2004, p.140).

\section{Notes}

1. See CAUL Best Practice website at http://www.caul.edu.au/best-practice/

2. See Final Report at http://www.caval.edu.au/wpr/crig/wp/\#WPPM

3. Wilson, A., Pitman L. \& Trahn, I. (2000). Guidelines for the application of best practice in Australian university libraries. Canberra: Dept. of Education, Training and Youth Affairs.

4. Wilson, A., \& Pitman, L. (2000). Best practice handbook for Australian university libraries. Canberra: Dept. of Education, Training and Youth Affairs.

5. See LTU Library Survey results at 
http://www.lib.latrobe.edu.au/about/surveys.php

6. See AUQA website at http://www.auqa.edu.au/

\section{References}

Association of College \& Research Libraries (ACRL). (2000). Standards for college libraries 2000 edition. Retrieved April 13, 2005, from http://www.ala.org/ala/acrl/acrlstandards/standardscollegelibraries.htm Association of Research Libraries (ARL). (1999). ARL New Measures Retreat January 14-15, 1999 Tucson, Arizona. Retrieved April 13, 2005 from http://www.arl.org/stats/program/transcript.html

Cribb, G., \& Hogan, C. (2003). Balanced Scorecard: linking strategic planning to measurement and communication. In: Proceedings of the 24th annual IATUL Conference, Ankara, Turkey. Paper retrieved April 13, 2005 from http://www.iatul.org/conference/proceedings/vol13/

Cullen, R. (2002). Setting standards for service quality. In J. Stein, M. Kyrillidou, $\&$ D. Davis (eds.): Meaningful measures for emerging realities: Proceedings of the 4th Northumbria International Conference on Performance Measurement in Library and Information Services (pp. 9-16). Washington, DC: Association of Research Libraries.

Hernon, P., \& Dugan, R. E. (2003). Different perspectives on assessment and evaluation: the need to refine and link them. In: Proceedings of the 5th Northumbria International Conference on Performance Measurement in Libraries and Information Services Durham, England.

Hiller, S., \& Self, J. (2004). From measurement to management: using data wisely for planning and decision-making. Library Trends, 53 (1), 129-155.

Lakos, A. (1998). Building a culture of assessment in academic libraries. Paper presented at a panel discussion at the Living the Future II Conference.

Tucson, AZ: University of Arizona, April 22, 1998.

Lakos, A. (1999). The missing ingredient - culture of assessment in libraries. Performance Measurement and Metrics, 1 (1), 3-7.

Lindauer, B. G., (1998). Defining and measuring the library's impact on campuswide outcomes. College \& Research Libraries, 59 (6), 78-102.

McGregor, F. (2000). Performance measures, benchmarking and value. In: Proceedings of the ALIA 2000 Conference, Canberra, Australia. Paper retrieved April 13, 2005 from http://www.alia.org.au/conferences/alia2000/proceedings/ felicity.mcgregor.html

McGregor, F. (2003). Benchmarking with the Best. In: Proceedings of the 5th Northumbria International Conference on Performance Measurement in Libraries and Information Services, Durham, England. 


\section{Appendix 1}

\section{LA TROBE UNIVERSITY LIBRARY QUALITY MANAGEMENT CHECKLIST}

1. Planning and Management

Planning

1.1 Does the Library have a strategic plan?

1.2 What is the mission?

1.3 What key factors internal and external to the University shape strategy?

1.4 Are objectives well aligned to those in the University and Faculty Strategic Plans?

1.5 Are staff and students involved in the development of these library plans?

1.6 Are library staff involved in the development? If yes, how is this achieved?

1.7 Do you regularly review the objectives and plans?

\section{Budgeting}

1.8 How do you ensure that resources follow strategy within the Library?

1.9 Are resource requirements prioritised and the budget regularly monitored to ensure that expenditure is kept within budget?

1.10 Is there a plan to replace computers and other equipment at appropriate intervals?

1.11 Are library staff consulted on budgetary issues? If yes, what is your decision making process on budgetary issues?

Communication

1.12 Are staff in the Library well informed about the University mission and objectives as well as those at library level? If yes, how do you provide this information?

1.13 How are library staff kept informed of changes in procedures?

1.14 How are library staff kept informed of decisions from elsewhere in the University which may impact on their activities?

1.15 How do your staff inform other areas of changes to policies, procedures and processes?

2. Staff

2.1 Do senior staff attend the Heads of Schools and Managers training programs conducted by the Personnel Office? 
2.2 Do staff have well defined position descriptions or duty statements?

2.3 Are there processes in place for induction of new members of staff to culture, policies and procedures? If yes, describe these processes.

2.4 Do you provide new staff and current staff with service standards or expectations for service levels? If yes, attach further information.

2.5 Does the Library encourage staff to enhance their skill base and/or qualifications? If yes, what is the process for achieving this?

2.6 How many staff have availed themselves of these opportunities?

2.7 Are there transparent workload allocation procedures?

2.8 Do you have a leave management strategy?

2.9 Are there procedures in place for dealing with staff concerns and difficulties? If yes, outline the procedures.

2.10 Is there a process for ensuring that PEDS supervision and reporting is up to date.

2.11 Is there a process for ensuring that IPPPS supervision and reporting is up to date.

2.12 If you have a multi campus operation is there some recognition of the implications of travel for workload, staff development opportunities?

3. Stakeholders and Standards

3.1 Do staff in the Library understand who your clientele are?

3.2 Do you know what services your customers/clients expect from you?

3.3 Are these expectations in line with your objectives?

3.4 Have staff in your area undertaken training in customer service principles?

3.5 Do you have standards set for service provision? E.g. turnaround times for services

3.6 Are these standards known to all staff in the library? If yes, how do you ensure this is communicated?

4. Policies and Processes

4.1 How do you achieve your objectives? ie identify the key procedures, structures, policies

4.2 Do you have some documentation of key processes/procedures?

4.3 Are these processes well understood by your staff ? If yes, how do you ensure this?

4.4 Have you recently reviewed procedures or practices? 
5. Community Engagement

5.1 Is there liaison with (as appropriate)

industry, community, government agencies, professional bodies, corresponding university libraries both in Australia and abroad, other educational institutions in Australia including secondary schools, TAFE institutes?

\section{Outcomes}

6.1 How do you know if you are achieving your objectives? What performance indicators or outcome measures do you use?

6.2 Are these measures closely matched to the objectives?

6.3 Do you seek customer feedback from the internal (staff and/or students) and external customers identified above? If yes, how do you do this?

7. Response

7.1 Have you identified any opportunities for improvement that are within the control of your unit?

7.2 What actions are planned for next year to address these improvement opportunities? What are the resource implications for these actions?

7.3 What strengths or successes have you identified?

7.4 Have these results been communicated to staff in your department? If yes, how has this been done?

7.5 Have you identified shortcomings in services or procedures that are outside the control of the library? What are your recommended responses?

October 2003

\section{Appendix 2}

\section{La Trobe University Library}

'Whole of Library' key measures and indicators - A Work in Progress

\section{General}

Selected indicators from CAUL annual statistics (e.g. salary expenditure as a \% of total expenditure)

Selected indicators from library annual statistics, primarily use measures (e.g. building use, number of reference transactions)

Customer satisfaction survey (Rodski survey) (satisfaction level, quality assessment, gaps between importance and performance)

Client feedback from print and electronic suggestion boxes 


\section{Services}

Information desk surveys (adequacy of support for library users)

Web usability survey (ease of access and use of electronic library services)

Information literacy evaluation (impact on user)

\section{Information Resources (Access and Availability)}

Materials availability survey (collection adequacy, shelving accuracy, catalogue error rate, library skills of user)

Intercampus loans and document delivery (turnaround time and fill rate)

Reshelving survey (turnaround time) (pilot project undertaken at one campus))

Order placement (turnaround time) (pilot project undertaken at one campus)

Electronic database usage statistics (under development)

\section{Facilities}

Study seating usage survey, including computer workstation usage (adequacy of study space)

Opening hours surveys (adequacy of access to the library building)

\section{Efficiency}

Unit cost (monograph and serial processing)

Intercampus loans and document delivery (turnaround time)

Reshelving (turnaround time)

Order placement (turnaround time)

\section{Abstract}

Australian university libraries have been implementing performance measurement, benchmarking and quality improvement initiatives over a long period of time. In recent years the systematic approach to evaluation, benchmarking and improvement has become an integral part of the Australian higher education framework as a whole. In 2000 the Australian government, which has overall responsibility for higher education in Australia, introduced a quality audit scheme and established the Australian Universities Quality Agency (AUQA). The AUQA audits focus on the goals and objectives of institutions, and investigate the processes universities have in place to achieve their goals and objectives, including the measuring, monitoring and improvement frameworks. The audits also include scrutiny of the institutions' libraries - how they contribute to institutional objectives and the quality assurance mechanisms in place to achieve this. This paper provides an overview of the key evaluation and measurement activities currently being developed and utilised by university libraries in Australia, 
and the higher education context which is catalysing these activities. Particular reference is paid to the work of the Council of Australian University Librarians (CAUL) in these areas, and the national benchmarking initiatives which are being undertaken. The paper also presents, as a case study, the preparation that one university library - La Trobe University Library - undertook for the AUQA quality audit, which was conducted in September 2004, including the "whole of library" performance measurement and evaluation activities which are being developed by the library to measure and monitor performance at the strategic level. 